\title{
HERRAMIENTAS PARA EL ANÁLISIS DE CAUSA RAIZ (ACR)
}

\section{TOOLS TO THE ROOT CAUSE ANALYSIS (RCA)}

\author{
Johanny del Carmen Ovalles Acosta ${ }^{1}$ \\ Víctor Gisbert Soler ${ }^{2}$ \\ Ana Isabel Pérez Molina ${ }^{3}$
}

1. Ingeniera Industrial. Pontificia Universidad Católica Madre y Maestra, República Dominicana. Máster Universitario en Organización y Logística. Universidad Politécnica de Valencia, España. E-mail: joov@epsa.upv.es

2. Doctor Ingeniero Industrial. Departamento de Estadística e Investigación Operativa Aplicadas y Calidad. Universidad Politécnica de Valencia, España. Email: vgisber@eio.upv.es

3. Ingeniera Técnico Industrial Química. Ingeniera en Organización Industrial. Doctor por la Universidad Politécnica de Valencia. Departamento de Estadística e Investigación Operativa Aplicadas y Calidad. Universidad Politécnica de Valencia, España. E-mail: anpemo@eio.upv.es

\section{Citación sugerida:}

Ovalles Acosta, J.C, Gisbert Soler, V. y Pérez Molina, A.I. (2017). Herramientas para el análisis de causa raíz (ACR). $3 C$ Empresa: investigación y pensamiento crítico, Edición Especial, 1-9. DOI: $<$ http://dx.doi.org/10.17993/3cemp.2017.especial.1-9/>. 


\section{RESUMEN}

Un método para solucionar problemas y que tiene la intención de eliminar, o en dado caso, disminuir la causa o las causas que los generan es el Análisis de Causa Raíz (ACR). Pero ¿por qué es tan importante identificar la causa de un problema? ¿Cuáles herramientas y técnicas se pueden utilizar para identificar dicha causa? ¿Qué efecto tiene mejorar de un proceso?

\section{ABSTRACT}

A method to solve problems and that has the intention to eliminate or decrease the root or the root causes that generates them is the Root Causa Analysis (Root Causa Analysis). But why is that important to identify the cause of a problem? Which tools and techniques can be used to identify that cause? What effect brings to improve a procees?

\section{PALABRAS CLAVE}

Problema, desviación, causa raíz, acción correctiva, acción preventiva, implementación y herramientas.

\section{KEY WORDS}

Problem, deviation, root cause, corrective action, preventive action, implementation and tools. 


\section{INTRODUCCIÓN}

Desde el inicio de los tiempos se ha ido arrastrando la necesidad de realizar actividades o procesos de una mejor manera, a fin de disminuir repeticiones, errores, defectos o simplemente para ahorrar tiempo y costes. Por este motivo se toma tiempo para analizar detalladamente, por medio de diferentes herramientas, la causa real que contribuye directamente a que una actividad falle o que no se desarrolle como deseamos para obtener los resultados esperados.

El Análisis de Causa Raíz (ACR) es básicamente una serie de metodologías que utilizan las organizaciones para establecer las causas que generan a determinadas cuestiones. El fin es utilizar el pensamiento objetivo para descifrar por qué algo salió mal o por qué algo no es posible, en lugar de culpar a los individuos o creer a los detractores que afirman que no se puede hacer o que se puede mejorar.

\section{ANTECEDENTES}

Con relación al surgimiento de las herramientas para realizar un análisis de causa raíz (ACR) tenemos al Dr. Kaoru Ishikawa el cual predicaba que la calidad debía ser llevada más allá del mismo trabajo, es decir, tenía que ser llevada a la vida diaria. Ishikawa hizo muchas aportaciones, además del diagrama de Causa-Efecto, entre las cuales demostró la importancia de las 7 herramientas de calidad y trabajó en los círculos de calidad. (Admindeempresas.blogspot.com.es, 2014)

Del mismo modo está Vilfredo Pareto, quien enunció por primera vez el principio de Pareto, el cual es conocido como la regla del 80-20, distribución A-B-C, ley de los pocos vitales o principio de escasez del factor, en el cual se elige la mejor acción a implementar, de acuerdo al evento presentado, ya que el $20 \%$ de los defectos afectan en el $80 \%$ de los procesos (Juran, 1975).

También Alex Faickney Osborn ideo la herramienta denominada como lluvia de ideas (brainstorming), la cual resultó en un proceso interactivo de grupo no estructurado que generaba más y mejores ideas que las que los individuos podían producir trabajando de forma independiente; dando oportunidad de dar sugerencias sobre un determinado asunto y aprovechando la capacidad creativa de los participantes (Stroebe, 1987). 


\section{HERRAMIENTAS PARA EL ANÁLISIS DE CAUSA RAIZ}

No todos los procesos de producción y provisión de servicios son perfectos, durante su ejecución se presentan eventos no conformes que los frenan o los desvían. Es necesario identificar correctamente la causa que originan las desviaciones en cada proceso de manera individual, a fin de poder implementar acciones correctivas y preventivas que las disminuyan o erradiquen completamente.

¿Por qué se necesita realizar un análisis de causa raíz?

Es necesario, ya que las organizaciones suelden responder a problemas con soluciones rápidas a medio término, sin embargo, depender de mejoras rápidas requiere que estas sean repetidas una y otra vez. Enfocarse en soluciones a corto plazo no es una receta para una mayor rentabilidad y crecimiento organizacional.

Para mejorar la eficiencia y rentabilidad, necesitamos observar más allá de la superficie de la raíz del problema o situación, observando el efecto (el cual solamente es el síntoma) de un problema, y deduciendo qué lo ha causado. Se pueden crear soluciones preventivas que deberían poner fin al problema, a fin de entender la fuente del problema, por lo tanto, se tendría que desarrollar un análisis de causa raíz.

Existen pautas para realizar un análisis de causa raíz, las cuales tienen el propósito de identificar la causa o las causas que inicia la generación de los eventos no conformes que atacan a proceso, estas pautas se enlistan a continuación:

- Identificar el problema

- Definir el problema

- Entender el problema

- Identificar la causa raíz

- Realizar acción correctiva

- Monitorear el sistema

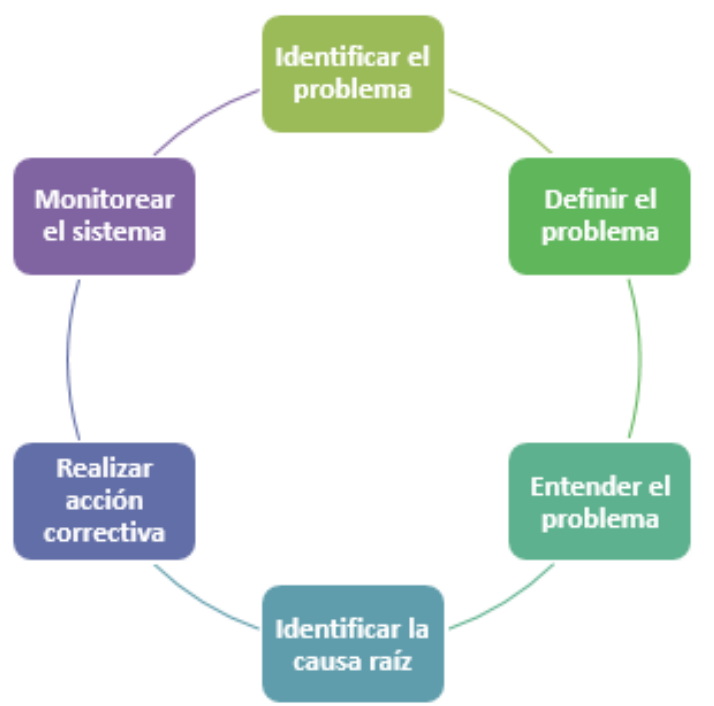

Figura 1. Pautas para realizar un Análisis de Causa Raiz (ACR). 
De los principales beneficios que tiene la eliminación de la causa raíz de un problema en el proceso, es que se puede ahorrar tiempo y dinero.

El primer paso para realizar un análisis de causa raíz, es identificar el problema. Es necesario monitorear varios aspectos de la organización, a fin de poder detectar posibles áreas problemáticas. El análisis de causa raíz se puede derivar de varias áreas que van desde queja de clientes, encuestas de clientes y notas de crédito de producto. Una vez el problema ha sido descubierto, existen cinco (5) pasos básicos para completar el Análisis de Causa Raíz (ACR).

A) Pasos básicos para completar el Análisis de Causa Raíz (ACR).

1. Definir el problema

2. Entender el problema

3. Implementar acción inmediata

4. Implementar acción correctiva

5. Confirmar la solución

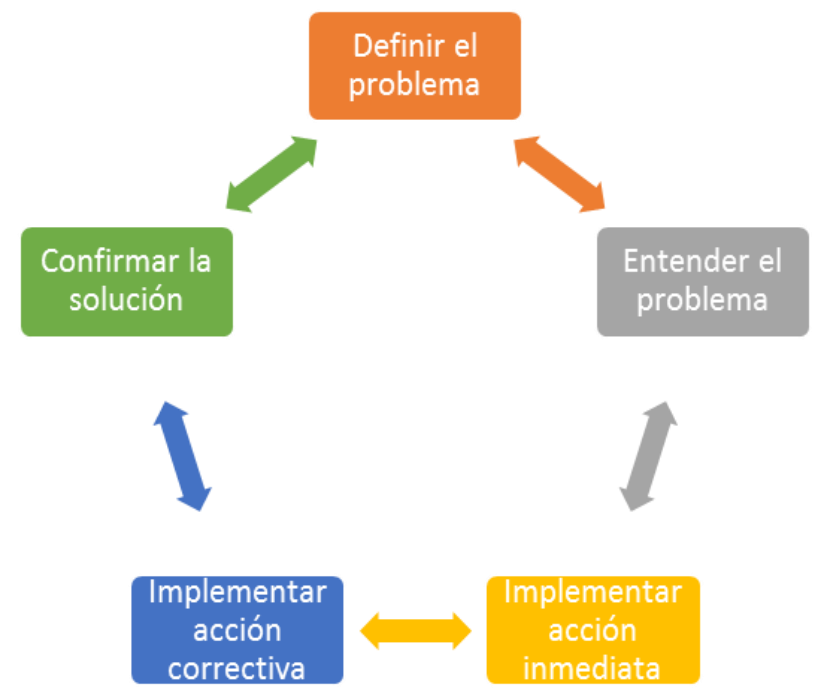

Figura 2. Pasos para completar el Analisis de Causa Raiz (ACR).

1- Definir el problema: Trata y utiliza el principio SMART (Specific, Measurable, Action oriented, Realistic, Time constrained). A menos de que el problema esté definido de manera precisa, todo el proceso de ARC, puede ser propenso al fracaso.

2- Entender el problema: Verifica la información, obteniendo información real relacionada al problema, ganando un claro entendimiento del problema. Es cuando las herramientas y técnicas, tanto como Causa y Efecto, lluvia de ideas, etc., pueden ser usadas. 
3- Acción inmediata: Implementar contramedidas temporales en el lugar del problema. Mientras más lejos se determine la solución de la fuente del problema, menos probable será que la solución sea efectiva.

4- Acción correctiva: Determinar y priorizar la causa más probable del problema, como las contramedidas temporales podrían no resolver la causa raíz. Tomar acciones correctivas para al menos mitigar o preferiblemente eliminar la o las causas.

5- Confirmar la solución: Después de que las medidas han sido determinadas e implementadas el éxito del enfoque adoptado necesita ser establecido. Habiendo confirmado el éxito de las soluciones sugeridas, entonces las reglas o métodos de control necesitan ser establecidos. Esta es probablemente la fase más importante del ACR, pero la que suele omitirse más.

B) Técnica de los Cinco (5) porqués

Los 5 porqués típicamente se refieren a la práctica de preguntar 5 veces por qué el fallo ha ocurrido, a fin de obtener la causa o las causas raíz del problema. Ninguna técnica especial o forma es requerida, pero los resultados deben ser capturados en una hoja de trabajo. Los 5 porqués es una excelente técnica para abordar un simple análisis de causa raíz (ACR).

C) Análisis de Pareto

El análisis de Pareto es una técnica fácil que ayuda a elegir el cambio o mejora más efectiva a implementar. El principio de Pareto o la regla 80/20, utiliza la idea de que el $20 \%$ de los defectos afectan en el $80 \%$ de los procesos. Por lo tanto, si las soluciones se enfocan al $20 \%$ de los problemas más relevantes que afectan a los procesos, es seguro que el $80 \%$ de los procesos mejoraran considerablemente.

D) Diagrama de Causa-Efecto

El diagrama de Causa-Efecto, también conocido como diagrama de espina de pescado o Ishikawa o análisis de $6 \mathrm{Ms}$, es una técnica bastante útil para realizar un análisis de causa raíz más compleja, profunda y detallada. Este tipo de diagrama identifica todos los potenciales factores que contribuyen a la generación de un problema en el proceso. En este diagrama se analizan factores como son los enlistados a continuación:

- Mano de Obra

- Método

- Máquina

- Material

- Medio ambiente

- Medición 


\section{E) Lluvia de ideas/Entrevistas}

Muchas personas están familiarizadas con la técnica de lluvia de ideas y entrevistas, sin embargo aquí se presentan algunos recordatorios:

- Recolecta tantas ideas como sea posible de parte de todos los participantes, sin criticar ni juzgar mientras las ideas son generadas.

- Todas las ideas son bienvenidas no importa que tontas o lejos parezcan. Se creativo, mientras más ideas mejor, porque en este punto no sabes que podría funcionar.

- Ninguna discusión secundaria debería de tomar lugar durante la lluvia de ideas, ya que el momento de discutirlas será al final cuando se haya completado la actividad.

- No criticar ni juzgar. Ni siquiera quejarse ni fruncir el ceño o reírse, ya que todas las ideas son iguales en este punto.

- No construyas en las ideas de otros.

- Escribe todas las ideas en una pizarra para que el grupo completo pueda visualizarlas, puedes usar el diagrama de Causa-Efecto para ayudar a capturar la información.

- Establece una hora límite para la lluvia de ideas. 30 minutos es casi suficiente. (MCQI, 2008)

\section{APLICACIÓN DE DIAGRAMA DE CAUSA-EFECTO}

En una línea de producción en la que se manufacturan productos médicos, se presentó una desviación durante el etiquetado del producto en proceso. Esta desviación consistía en que las etiquetas se estaban adhiriendo a la unidad de manera incorrecta (etiquetas rotadas y desalineadas), esta situación generó un incremento de unidades defectuosas en la línea de producción, lo que no permitió que el producto terminado llegara a tiempo al cliente y se tuviera que re trabajar dicho producto, desperdiciando tiempo, mano de obra, materiales y otros costes.

Para mitigar el problema se utilizó, en este caso, el diagrama de Causa-Efecto o análisis de 6 Ms (Mano de Obra, Método, Máquina, Material, Medio ambiente y Medición), a fin de poder identificar la causa raíz que generó el problema y poder implementar acciones para corregir el mismo. Luego de analizar todas las Ms, se identificó que al colocar en el transportador (conveyor) las unidades a etiquetar, estas se movían de posición, debido a la vibración provocada por el transportador (conveyor) al estar encendido, provocando desalineación de las unidades y por ende unidades con etiquetas rotadas o desalineadas. Se identificó que la M que contribuyó a que se generara esta situación fue la M de Máquina. 
La acción que se implementó para corregir dicha situación fue la colocación de varios moldes con la forma de las unidades (Poka Yokes) en varios puntos clave del conveyor, los cuales no permitían que pasaran unidades rotadas por la vibración del conveyor. Dicha acción contribuyó a que las unidades a etiquetar se tuvieran que alinear manualmente o por medio de la vibración para que las unidades pudieran pasar por el poka yoke y de esta forma que lleguen alineadas a la etiquetadora y pueden ser etiquetadas con la posición correcta de las unidades, disminuyendo de esta forma la cantidad de unidades etiquetadas fuera de especificación.

\section{CONCLUSIONES}

Se desarrolló el análisis de Causa-Efecto, por lo que se pudo identificar y atacar la causa raíz que provocaba en la línea de producción la generación de unidades defectuosas por etiquetado fuera de especificación (etiquetas rotadas y desalineadas). Por lo tanto, se puede concluir en que luego de implementar las acciones correctivas descritas anteriormente se pudo tener una línea de producción más eficiente y con menos unidades defectuosas.

Del mismo modo las acciones correctivas contribuyeron a que haya un flujo de unidades menos interrumpido, a que se disminuyeran los re trabajos, los que generaba un incremento en el coste de mano de obra, coste de materiales, entre otros costes indirectos. Por lo tanto, una vez más se pudo evidenciar la importancia del uso de las herramientas de Análisis de Causa Raíz (ACR), confirmando sus aportes a la resolución de problemas sin importar el área o sector donde estos emerjan. Igualmente, dichas herramientas dirigen a la implementación de mejoras que mejor se ajusten para la erradicación de una desviación en algún proceso. 


\section{REFERENCIAS BIBLIOGRÁFICAS}

- http://admindeempresas.blogspot.com.es/2014/10/biografia-y-principalesaportaciones.html

- Juran, Joseph (1975) The Non-Pareto Principle; Mea Culpa

- Michael Diehl y Wolfgang Stroebe (1987). «Productivity Loss in Brainstorming Groups: Toward the Solution of a Riddle

- https://books.google.es/books?id=dgqyBQAAQBAJ\&pg=PA88\&dq=analisis+de+caus a+raiz+lean\&hl=es\&sa=X\&ved=0ahUKEwiZsvvWgujTAhWDORoKHVz-

B4AQ6AEIJzAA\#v=onepage $\& q=$ analisis\%20de\%20causa\%20raiz\%20lean \& $f=$ false

- http://www.root-cause-

analysis.co.uk/images/Green\%20RCA\%20mini\%20guide\%20v5\%20small.pdf 